HETEROCYCLES, Vol. 71, No. 10, 2007, pp. 2203 - 2210. @ The Japan Institute of Heterocyclic Chemistry Received, 21st May, 2007, Accepted, 3rd July, 2007, Published online, 17th July, 2007. COM-07-11113

\title{
NOVEL AND EFFICIENT SYNTHESIS OF 5,8-DIMETHYL-9H- CARBAZOL-3-OL VIA A HYDROXYDEBORONATION REACTION
}

Anna Caruso, ${ }^{\mathrm{a}, \mathrm{b}}$ Anne Sophie Voisin-Chiret, ${ }^{\mathrm{a}}$ Jean-Charles Lancelot, ${ }^{\mathrm{a}}$ Maria Stefania Sinicropi, ${ }^{\mathrm{b}}$ Antonio Garofalo, ${ }^{\mathrm{b}}$ and Sylvain Rault ${ }^{\mathrm{a}, *}$

${ }^{a}$ Université de Caen Basse-Normandie, U.F.R. des Sciences Pharmaceutiques, Centre d'Etudes et de Recherche sur le Médicament de Normandie, 14032 Caen, France, ${ }^{\mathrm{b} D i p a r t i m e n t o}$ di Scienze Farmaceutiche, Università della Calabria, 87036 Arcavacata di Rende, (Cs), Italy

*Corresponding author: tel. +3323193 4169; fax +3323193 1188; e-mail sylvain.rault@unicaen.fr

\begin{abstract}
N$-Protected carbazol-3-yl-boronic acid derivatives have been efficiently hydroxydeboronated under mild conditions by employing hydrogen peroxide. The method allows to easily obtain 3-hydroxycarbazoles as precursors of new analogs of the anticancer agent 9-hydroxyellipticine.
\end{abstract}

\section{INTRODUCTION}

Since thirty years, Ellipticine (5,11-dimethyl-6H-pyrido[4,3-b]carbazole) (I) (Chart 1) is a well known alkaloid with antitumor properties, acting as a DNA intercalating agent and inhibiting the activity of topoisomerase II. Currently, recent works demonstrated that Ellipticine induces apoptotic cell death by the generation of cytotoxic free radicals and interestingly, a recent study by Hagg et al. ${ }^{1 \mathrm{~g}}$ reported that Ellipticine mediated apoptosis induced by endoplasmic reticulum stress. ${ }^{1}$ However, some limitations, such as low water solubility and cardiovascular side effects, preclude its therapeutic use. Many structural modifications on the original molecule have been designed in order to obtain several derivatives with a better pharmacological profile., 


\section{Chart 1}

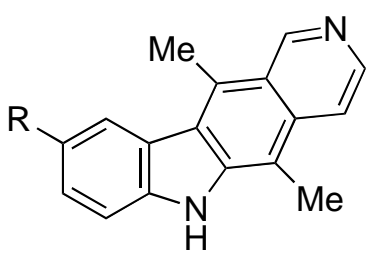

(I) $\mathrm{R}=\mathrm{H}$

(II) $\mathrm{R}=\mathrm{OH}$

In particular, 9-hydroxyellipticine, Celiptium ${ }^{\circledR}$, (II) (Chart 1) showed to possess a higher DNA affinity than ellipticine itself, high activity on L1210 mice leukaemia, and lack of toxicity at therapeutic doses. ${ }^{4}$ The introduction of the hydroxy group in position 9 would strenghten the interaction with the DNA through the formation of a hydrogen bond with the negatively charged oxygen of a phosphate group. The preparation of 9-hydroxy derivatives of ellipticine is tightly related with 5,8-dimethyl-9H-carbazol-3-ol (III) (Chart 2), which were previously obtained by a quite demanding synthetic route using 5methoxyindole, a very expensive starting material. ${ }^{5}$ Herein, we describe a convenient alternative method for the synthesis of compounds III as precursors of new analogs of II.

\section{Chart 2}<smiles>Cc1ccc(C)c2c1[nH]c1ccc(O)cc12</smiles>

(III)

The key step of the process consists in the conversion of $\mathrm{N}$-protected 5,8-dimethyl-9H-carbazol-3-ylboronic acids into the corresponding 3-hydroxy derivatives using hydrogen peroxide in mild reaction conditions. ${ }^{6}$ The suitable boronic acids were in turn prepared starting from 5-bromoindole by a smooth multi-step process, going through the formation of corresponding boronic dimethyl esters. This procedure allows also the preparation of the boronic pinacolyl ester but all attempts to apply a similar hydroxydeboronation with this boronic pinacolyl ester failed. The method allows the preparation of 9unsubstituted 5,8-dimethyl-9H-carbazol-3-ols when the easily removable $N$-protecting Boc group was used.

\section{RESULTS AND DISCUSSION}

The new synthetic pathway to 5,8-dimethyl-9H-carbazol-3-ols is described in the Scheme. The starting 6bromo-1,4-dimethyl-9H-carbazole (1) was prepared by reaction of 5-bromoindole with hexane-2,4-dione 
in the presence of $p$-toluenesulphonic acid. ${ }^{7-9}$ This starting compound was $N$-protected by (Boc) ${ }_{2} \mathrm{O}$ to give compound $\mathbf{2} \mathbf{a}^{10}$ or, alternatively, $N$-methylated by iodomethane and sodium hydride under standard conditions to give compound $\mathbf{2 b} .{ }^{11}$ Compounds $\mathbf{2 a}$ and $\mathbf{2 b}$ were subjected to lithiation-boronation at very low temperature, using $n$-BuLi and trimethyl borate after an usual hydrolytic work-up to give the corresponding 3-boronic acid derivatives $\mathbf{3 a}$ and $\mathbf{3 b}$, respectively. ${ }^{6,12-15}$

$\mathrm{N}$-Boc-5,8-dimethyl-9H-carbazol-3-boronic acid (3a) was first treated with an aqueous mixture of bases $\left(\mathrm{NaOH}\right.$ and $\left.\mathrm{NaHCO}_{3}\right)$ at $50^{\circ} \mathrm{C}$, then hydrogen peroxide was added to the reaction mixture at room temperature.

The expected 3-hydroxy derivative $4 \mathbf{a}$ was obtained in $82 \%$ yield by a final moderate acidification (1N $\mathrm{HCl}, \mathrm{pH} 4)$. In the same way, compound $\mathbf{3 b}$ was converted into 3 -hydroxy derivative $\mathbf{4 b}$ in $70 \%$ yield. The corresponding pinacolyl ester 3c, obtained after a similar fashion to that described for compounds 3a, but using tri-i-propyl borate and pinacol instead of trimethyl borate, proved to be unreactive under similar reaction conditions.

Finally, Boc protecting group of compound $\mathbf{4 a}$ was removed by hydrolytic treatment (6N HCl, pH 1), after the hydroxydeboronation step, to give 5,8-dimethyl-9H-carbazol-3-ol (5) in 75\% yield. The attempt to directly perform the hydroxydeboronation reaction on the previously $N$-deprotected boronic acid $3 \mathbf{d}$ also led to compound $\mathbf{5}$, but in a considerably lower yield.

Scheme $^{\mathrm{a}}$

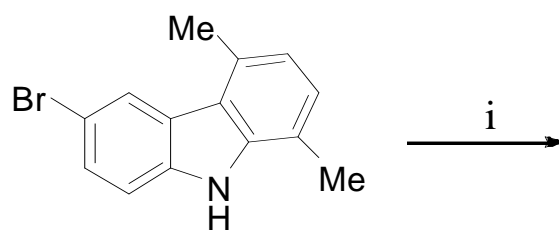

1

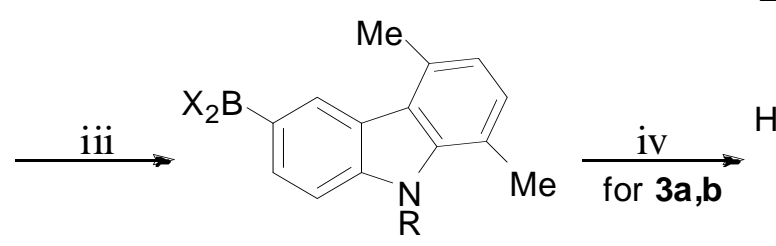

3a : $\mathbf{R}=\mathrm{BOc}, \mathrm{X}=\mathrm{OH}$

$3 \mathrm{~b}: \mathrm{R}=\mathrm{Me}, \mathrm{X}=\mathrm{OH}$

3c: $\mathbf{R}=\mathrm{Me}, \mathrm{X}_{2}=$ pinacolyl

3d : R = H, X = OH

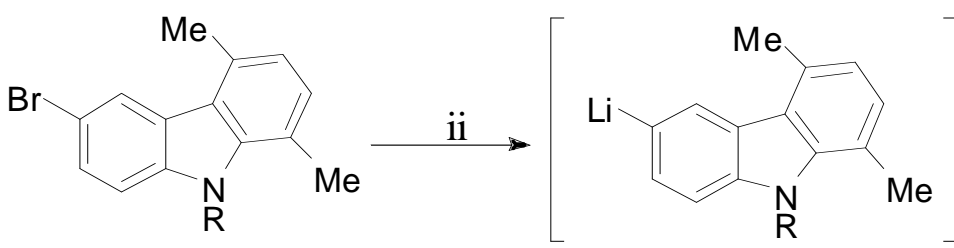

2a : R = Boc

$2 b: R=M e$ 


\section{EXPERIMENTAL}

Melting points were determined on a Kofler melting point apparatus and are uncorrected. IR spectra were taken with a Perkin Elmer BX FT-IR. HRMS (EI) determinations were made using a spectrometer JEOL JMS GCMate. ESI-MS was performed using a spectrometer LC-MS Waters alliance 2695 (ESI+). ${ }^{1} \mathrm{H}-$ NMR (400 MHz) and ${ }^{13} \mathrm{C}-\mathrm{NMR}(100 \mathrm{MHz})$ were recorded on a JEOL Lambda 400 Spectrometer. Chemical shifts are expressed in parts per million downfield from tetramethylsilane as an internal standard. Thin layer chromatography (TLC) was performed on silica gel 60F-264 (Merck). Elemental analyses were performed at the 'Institut de Recherche en Chimie Organique Fine' (Rouen).

\section{6-Bromo-1,4-dimethyl-9H-carbazole (1)}

A suspension of 5-bromoindole $(5.0 \mathrm{~g}, 25.0 \mathrm{mmol})$, hexane-2,4-dione $(3.5 \mathrm{~g}, 31.0 \mathrm{mmol})$ and $p$ toluenesulphonic acid $(3.86 \mathrm{~g}, 20.0 \mathrm{mmol})$ in absolute EtOH $(200 \mathrm{~mL})$ was refluxed for $4 \mathrm{~h}$ and then left overnight at rt. The resulting solution was diluted with water $(400 \mathrm{~mL})$ and $\mathrm{Et}_{2} \mathrm{O}(800 \mathrm{~mL})$. The organic layer was separated and shaken with saturated aqueous $\mathrm{NaHCO}_{3}$ until neutrality then dried and evaporated to give pure compound 1 as a purple solid (4.9 g, 70\% yield), mp $134{ }^{\circ} \mathrm{C}$ (EtOAc/hexanes). IR (KBr): $3407,1447,1297,811,797,537 \mathrm{~cm}^{-1} .{ }^{1} \mathrm{H}$ NMR (DMSO-d $) \delta 11.36$ (s, 1H, NH); 8.17 (s, 1H, H5); 7.51$7.48(\mathrm{~m}, 2 \mathrm{H}, \mathrm{H} 7-\mathrm{H} 8) ; 7.11\left(\mathrm{~d}, \mathrm{~J}_{\mathrm{H} 2-3}=7.32 \mathrm{~Hz}, 1 \mathrm{H}, \mathrm{H} 2\right) ; 6.87$ (d, $\left.\mathrm{J}_{\mathrm{H} 3-2}=7.32 \mathrm{~Hz}, 1 \mathrm{H}, \mathrm{H} 3\right) ; 2.77$ (s, 3H, $\left.\mathrm{CH}_{3}\right) ; 2.54\left(\mathrm{~s}, 3 \mathrm{H}, \mathrm{CH}_{3}\right)$.

\section{6-Bromo-9-tert-butoxycarbonyl-1,4-dimethyl-9H-carbazole (2a)}

To a solution of 6-bromo-1,4-dimethyl-9H-carbazole (1) $(5.0 \mathrm{~g}, 18.2 \mathrm{mmol})$ in $\mathrm{MeCN}(70 \mathrm{~mL})$ cooled to $0{ }^{\circ} \mathrm{C}$ were added $\mathrm{Et}_{3} \mathrm{~N}(5.07 \mathrm{~mL}, 36.5 \mathrm{mmol})$, DMAP $(4.46 \mathrm{~g}, 36.5 \mathrm{mmol})$ and di-tert-butyl dicarbonate (7.96 g, $36.5 \mathrm{mmol})$. The mixture was stirred for $1 \mathrm{~h}$ at $0{ }^{\circ} \mathrm{C}$, then left at $\mathrm{rt}$ for $3 \mathrm{~h}$. The residue obtained after removal of the solvent was diluted with EtOAc $(100 \mathrm{~mL})$ and shaken with water $(2 \mathrm{x} 100 \mathrm{~mL})$. The residue obtained after an usual work-up was purified by silica gel column chromatography using cyclohexane/ $\mathrm{Et}_{2} \mathrm{O}(7: 3)$ as eluent to give compound $\mathbf{2 a}$ as a yellow solid (4.3 g, 63\% yield), mp $112{ }^{\circ} \mathrm{C}$. IR (KBr): 3446, 2971, 1738, 1448, 1368, 1296, 1248, 1153, 794, $535 \mathrm{~cm}^{-1} .{ }^{1} \mathrm{H}$ NMR (DMSO-d $d_{6} \delta 8.24(\mathrm{~d}$, $\left.\mathrm{J}_{\mathrm{H} 5-7}=1.96 \mathrm{~Hz}, 1 \mathrm{H}, \mathrm{H} 5\right) ; 7.99\left(\mathrm{dd}, \mathrm{J}_{\mathrm{H} 7-8}=8.80 \mathrm{~Hz}, 1 \mathrm{H}, \mathrm{H} 7\right) ; 7.74\left(\mathrm{dd}, \mathrm{J}_{\mathrm{H} 8-7}=8.80 \mathrm{~Hz}, 1 \mathrm{H}, \mathrm{H} 8\right) ; 7.29(\mathrm{~d}$, $\left.\mathrm{J}_{\mathrm{H} 2-3}=7.80 \mathrm{~Hz}, 1 \mathrm{H}, \mathrm{H} 2\right) ; 7.20\left(\mathrm{~d}, \mathrm{~J}_{\mathrm{H} 3-2}=7.80 \mathrm{~Hz}, 1 \mathrm{H}, \mathrm{H} 3\right) ; 2.81\left(\mathrm{~s}, 3 \mathrm{H}, \mathrm{CH}_{3}\right) ; 2.55\left(\mathrm{~s}, 3 \mathrm{H}, \mathrm{CH}_{3}\right) ; 1.73(\mathrm{~s}$, $\left.9 \mathrm{H}, \mathrm{CH}_{3}\right) .{ }^{13} \mathrm{C}$ NMR (DMSO- $\left.d_{6}\right) \delta 149.8,138.1,138.0,130.3,130.1,129.0,127.8,125.5,124.4,123.2$,

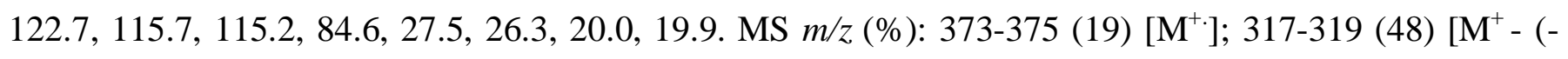
$t \mathrm{Bu})] ; 273-275(100)\left[\mathrm{M}^{+}-\left(-\mathrm{CO}_{2} t \mathrm{Bu}\right)\right] ; 193(52)\left[\mathrm{M}^{+}-\left(\mathrm{Br},-\mathrm{CO}_{2} t \mathrm{Bu}\right)\right] ; 81$ (39). Anal. Calcd for $\mathrm{C}_{19} \mathrm{H}_{20} \mathrm{BrNO}_{2}$ : C, 60.97; H, 5.39; N, 3.74. Found: C, 60.75; H, 5.08; N, 3.51. 
A mixture of 6-bromo-1,4-dimethyl-9H-carbazole (1) $(2.0 \mathrm{~g}, 7.3 \mathrm{mmol})$ and dry DMF $(60 \mathrm{~mL})$ was stirred at $\mathrm{rt}$ until clear. $\mathrm{NaH} 60 \%$ oil dispersion $(0.44 \mathrm{~g}, 10.9 \mathrm{mmol})$ and was added at $0{ }^{\circ} \mathrm{C}$. After $15 \mathrm{~min}$ stirring, iodomethane $(1.46 \mathrm{~mL}, 21.9 \mathrm{mmol})$ was added and the mixture was stirred for $1 \mathrm{~h}$ at $\mathrm{rt}$. Water $(200 \mathrm{~mL})$ was then added and the resulting mixture was extracted with EtOAc $(2 \times 200 \mathrm{~mL})$. Removal of the solvent gave pure compound $\mathbf{2 b}$ as a white solid (2.0 g, 95\% yield), mp $138{ }^{\circ} \mathrm{C}$ (EtOAc/hexanes). IR (KBr): 3435, 2921, 1469, 1296, 1092, 791, $533 \mathrm{~cm}^{-1} .{ }^{1} \mathrm{H}$ NMR (DMSO- $\left.d_{6}\right) \delta 8.18$ (d, J $\left.\mathrm{H}_{\mathrm{H}-7}=1.48 \mathrm{~Hz}, 1 \mathrm{H}, \mathrm{H} 5\right)$; 7.617.55 (m, 2H, H7-H8); 7.10 (d, J $\left.\mathrm{H}_{\mathrm{H}-3}=7.32 \mathrm{~Hz}, 1 \mathrm{H}, \mathrm{H} 2\right) ; 6.88$ (d, J $\left.\mathrm{J}_{\mathrm{H} 3-2}=7.32 \mathrm{~Hz}, 1 \mathrm{H}, \mathrm{H} 3\right) ; 4.10$ (s, 3H, $\mathrm{NCH}_{3}$ ); 2.79 (s, 3H, CH $\left.\mathrm{CH}_{3}\right) ; .72$ (s, 3H, $\left.\mathrm{CH}_{3}\right) .{ }^{13} \mathrm{C} \mathrm{NMR}\left(\mathrm{DMSO}-\mathrm{d}_{6}\right) \delta 140.0,139.7,130.8,129.3,127.4$, 124.4, 124.1, 121.1, 120.2, 118.5, 111.2, 110.9, 32.4, 20.5, 19.8. MS m/z (\%): 287 (100) $\left[\mathrm{M}^{+}\right]$; 272 (25) $\left[\mathrm{M}^{+}-\left(-\mathrm{CH}_{3}\right)\right] ; 193(24)\left[\mathrm{M}^{+}-\left(\mathrm{Br},-\mathrm{CH}_{3}\right)\right]$. Anal. Calcd for $\mathrm{C}_{15} \mathrm{H}_{14} \mathrm{BrN}$ : C, 62.52; H, 4.90; N, 4.86. Found: C, 62.67; H, 5.13; N, 4.63.

\section{9-tert-Butoxycarbonyl-5,8-dimethyl-9H-carbazole-3-boronic acid (3a)}

To a solution of the bromoderivative $2 \mathbf{a}(3.7 \mathrm{~g}, 10.0 \mathrm{mmol})$ in anhydrous THF $(250 \mathrm{~mL})$ cooled to $-90{ }^{\circ} \mathrm{C}$ was added dropwise a solution of $2.5 \mathrm{M} \mathrm{n}$-BuLi in hexanes $(4.8 \mathrm{~mL}, 12.0 \mathrm{mmol})$. The mixture was allowed to react at $-85^{\circ} \mathrm{C}$ for $1 \mathrm{~h}$. Trimethyl borate $(1.2 \mathrm{~mL}, 11.0 \mathrm{mmol})$ was added dropwise at the same temperature. The resulting solution was stirred for an additional $1 \mathrm{~h}$ then it was acidified to $\mathrm{pH} 4 \mathrm{by}$ dropwise addition of $0.5 \mathrm{~N} \mathrm{HCl}$ at $-30{ }^{\circ} \mathrm{C}$. The mixture was allowed to warm to rt then poured into $\mathrm{H}_{2} \mathrm{O}$ $(300 \mathrm{~mL}) . \mathrm{Et}_{2} \mathrm{O}(2 \times 250 \mathrm{~mL})$ extraction gave pure compound 3a as a white solid (2.0 g, $60 \%$ yield $)$, $\mathrm{mp}$ $250{ }^{\circ} \mathrm{C}(\mathrm{EtOH}) . \mathrm{IR}(\mathrm{KBr}): 3435,2975,1738,1607,1434,1334,1246,1152,1080,801,716 \mathrm{~cm}^{-1} .{ }^{1} \mathrm{H}$ $\mathrm{NMR}\left(\mathrm{CD}_{3} \mathrm{OD}\right) \delta 8.40(\mathrm{~s}, 1 \mathrm{H}, \mathrm{H} 5) ; 8.03\left(\mathrm{~d}, \mathrm{~J}_{\mathrm{H} 7-8}=8.56 \mathrm{~Hz}, 1 \mathrm{H}, \mathrm{H} 7\right) ; 7.73\left(\mathrm{~d}, \mathrm{~J}_{\mathrm{H} 8-7}=8.56 \mathrm{~Hz}, 1 \mathrm{H}, \mathrm{H} 8\right)$; $7.15\left(\mathrm{~d}, \mathrm{~J}_{\mathrm{H} 2-3}=7.56 \mathrm{~Hz}, 1 \mathrm{H}, \mathrm{H} 2\right) ; 7.07\left(\mathrm{~d}, \mathrm{~J}_{\mathrm{H} 3-2}=7.56 \mathrm{~Hz}, 1 \mathrm{H}, \mathrm{H} 3\right) ; 2.77\left(\mathrm{~s}, 3 \mathrm{H}, \mathrm{CH}_{3}\right) ; 2.44\left(\mathrm{~s}, 3 \mathrm{H}, \mathrm{CH}_{3}\right)$; $1.74\left(\mathrm{~s}, 9 \mathrm{H}, \mathrm{CH}_{3}\right) .{ }^{13} \mathrm{C} \mathrm{NMR}\left(\mathrm{CD}_{3} \mathrm{OD}\right) \delta 150.9,139.8,132.5,131.1,130.5,128.7,127.5,126.2,126.1$, 124.1, 123.1, 114.8, 85.0, 28.3, 20.7. MS m/z (\%): 295 (12) $\left[\mathrm{M}^{+}-\left(-\mathrm{B}(\mathrm{OH})_{2}\right)\right] ; 239(21)\left[\mathrm{M}^{+}-(-t \mathrm{Bu})\right] ; 195$ (100) $\left[\mathrm{M}^{+}-\left(-\mathrm{B}(\mathrm{OH})_{2},-\mathrm{CO}_{2} t \mathrm{Bu}\right)\right]$. Anal. Calcd for $\mathrm{C}_{19} \mathrm{H}_{22} \mathrm{BNO}_{4}: \mathrm{C}, 67.28 ; \mathrm{H}, 6.54 ; \mathrm{N}, 4.13$. Found: $\mathrm{C}$, 67.49; H, 6.32; N, 4.29.

\section{5,8,9-Trimethyl-9H-carbazole-3-boronic acid (3b)}

Following a procedure identical to that described for compound $\mathbf{3 a}$, but using compound $\mathbf{2 b}$ (2.9 g, 10.0 mmol) as starting material, compound $\mathbf{3 b}$ was obtained as a white solid (1.3 g, $50 \%$ yield), $\mathrm{mp}>270{ }^{\circ} \mathrm{C}$ (EtOH). IR (KBr): 3426, 2923, 1611, 1348, 1251, 1094, 796, $711 \mathrm{~cm}^{-1} .{ }^{1} \mathrm{H}$ NMR (DMSO-d $) \delta 8.61(\mathrm{~s}$, $1 \mathrm{H}, \mathrm{H} 5) ; 7.89$ (d, $\left.\mathrm{J}_{\mathrm{H} 7-8}=8.32 \mathrm{~Hz}, 1 \mathrm{H}, \mathrm{H} 7\right) ; 7.84(\mathrm{~s}, 2 \mathrm{H}, \mathrm{OH}) ; 7.51$ (d, $\left.\mathrm{J}_{\mathrm{H} 8-7}=8.32 \mathrm{~Hz}, 1 \mathrm{H}, \mathrm{H} 8\right) ; 7.04$ (d, $\left.\mathrm{J}_{\mathrm{H} 2-3}=7.56 \mathrm{~Hz}, 1 \mathrm{H}, \mathrm{H} 2\right) ; 6.85\left(\mathrm{~d}, \mathrm{~J}_{\mathrm{H} 3-2}=7.56 \mathrm{~Hz}, 1 \mathrm{H}, \mathrm{H} 3\right) ; 4.10\left(\mathrm{~s}, 3 \mathrm{H}, \mathrm{NCH}_{3}\right) ; 2.80\left(\mathrm{~s}, 3 \mathrm{H}, \mathrm{CH}_{3}\right), 2.79(\mathrm{~s}$, $\left.3 \mathrm{H}, \mathrm{CH}_{3}\right)$. MS m/z (\%): 209 (100) $\left[\mathrm{M}^{+}-\left(-\mathrm{B}(\mathrm{OH})_{2}\right)\right] ; 194(46)\left[\mathrm{M}^{+}-\left(\mathrm{CH}_{3},-\mathrm{B}(\mathrm{OH})_{2}\right)\right]$. Anal. Calcd for $\mathrm{C}_{15} \mathrm{H}_{16} \mathrm{BNO}_{2}$ : C, 71.18; H, 6.37; N, 5.53. Found: C, 70.90; H, 6.28; N, 5.43. 


\section{5,8-Dimethyl-9H-carbazole-3-boronic acid (3d)}

Following a procedure identical to that described for compound 3a, but reaching $\mathrm{pH} 1$ by the use of $6 \mathrm{~N}$ $\mathrm{HCl}$ for the hydrolytic work-up, compound 3d was obtained as a white solid (2.1 g, 90\% yield), mp > $260{ }^{\circ} \mathrm{C}(\mathrm{EtOH}) . \mathrm{IR}(\mathrm{KBr}): 3214,1567,1418,1257,867,719,546 \mathrm{~cm}^{-1} .{ }^{1} \mathrm{H}$ NMR (DMSO-d $d_{6} \delta 8.57(\mathrm{~s}$, 1H, H5); 8.16 (s, 3H, OH, NH); 7.94-7.93 (m, 2H, H7-8); 7.18 (d, J J $=7.56 \mathrm{~Hz}, 1 \mathrm{H}, \mathrm{H} 3) ; 2.78\left(\mathrm{~s}, 3 \mathrm{H}, \mathrm{CH}_{3}\right) ; 2.40\left(\mathrm{~s}, 3 \mathrm{H}, \mathrm{CH}_{3}\right)$. Anal. Calcd for $\mathrm{C}_{14} \mathrm{H}_{14} \mathrm{BNO}_{2}$ : C, 70.33; H, 5.90; N, 5.86. Found: C, 70.00; H, 5.70; N, 5.80.

\section{1,4,9-Trimethyl-6-(4,4,5,5-tetramethyl-1,3,2-dioxaborolan-2-yl)-9H-carbazole (3c)}

To a solution of the bromoderivative $2 \mathbf{b}(1.0 \mathrm{~g}, 3.5 \mathrm{mmol})$ in anhydrous THF $(100 \mathrm{~mL})$ cooled to $-90{ }^{\circ} \mathrm{C}$ was added dropwise a solution of $2.5 \mathrm{M} n$-BuLi in hexanes $(1.8 \mathrm{~mL}, 4.5 \mathrm{mmol})$. The mixture was allowed to react at $-85{ }^{\circ} \mathrm{C}$ for $1 \mathrm{~h}$. Triisopropyl borate $(0.9 \mathrm{~mL}, 3.9 \mathrm{mmol})$ was added dropwise at the same temperature. The resulting solution was stirred for an additional $1 \mathrm{~h}$, then warmed to $0^{\circ} \mathrm{C}$ and a solution of pinacol $(0.41 \mathrm{~g}, 3.5 \mathrm{mmol})$ in anhydrous THF $(7 \mathrm{~mL})$ was added slowly. The mixture was allowed to warm at $\mathrm{rt}$ then it was acidified to $\mathrm{pH} 4$ by dropwise addition of acetic acid. $\mathrm{Et}_{2} \mathrm{O}(2 \times 150 \mathrm{~mL})$ extraction gave pure compound $\mathbf{3 c}$ as a white solid (0.9 g, 77\% yield), $\mathrm{mp}>270{ }^{\circ} \mathrm{C}$. IR (KBr): 3434, 2924, 1611, 1353, 1253, 1149, 1098, 878, 801, 785, $679 \mathrm{~cm}^{-1} .{ }^{1} \mathrm{H}$ NMR (DMSO- $\left.d_{6}\right) \delta 8.43(\mathrm{~s}, 1 \mathrm{H}, \mathrm{H} 5) ; 7.75\left(\mathrm{~d}, \mathrm{~J}_{\mathrm{H} 7-8}=\right.$ $8.32 \mathrm{~Hz}, 1 \mathrm{H}, \mathrm{H} 7) ; 7.58\left(\mathrm{~d}, \mathrm{~J}_{\mathrm{H} 8-7}=8.32 \mathrm{~Hz}, 1 \mathrm{H}, \mathrm{H} 8\right) ; 7.07$ (d, $\left.\mathrm{J}_{\mathrm{H} 2-3}=7.32 \mathrm{~Hz}, 1 \mathrm{H}, \mathrm{H} 2\right) ; 6.88\left(\mathrm{~d}, \mathrm{~J}_{\mathrm{H} 3-2}=7.32\right.$ $\mathrm{Hz}, 1 \mathrm{H}, \mathrm{H} 3) ; 4.12\left(\mathrm{~s}, 3 \mathrm{H}, \mathrm{NCH}_{3}\right) ; 2.80\left(\mathrm{~s}, 3 \mathrm{H}, \mathrm{CH}_{3}\right) ; 2.74\left(\mathrm{~s}, 3 \mathrm{H}, \mathrm{CH}_{3}\right) ; 1.32\left(\mathrm{~s}, 12 \mathrm{H}, \mathrm{CH}_{3}\right) .{ }^{13} \mathrm{C} \mathrm{NMR}$ $\left(\right.$ DMSO$\left._{-}{ }_{6}\right) \delta 143.2,139.2,131.1,130.1,128.6,128.5,121.2,120.9,118.1,118.0,108.4,108.3,83.2,81.1$, 32.1, 24.7, 24.5, 20.4, 19.6. MS m/z (\%): 335 (100) [M $\left.\mathrm{M}^{+}\right]$; 209 (51). Anal. Calcd for $\mathrm{C}_{21} \mathrm{H}_{26} \mathrm{BNO}_{2}: \mathrm{C}$, 75.24; H, 7.82; N, 4.18. Found: C, 75.08; H, 7.69; N, 4.02.

\section{tert-Butyl 6-hydroxy-1,4-dimethyl-9H-carbazole-9-carboxylate (4a)}

A mixture of boronic acid 3a $(1.6 \mathrm{~g}, 4.8 \mathrm{mmol}), \mathrm{H}_{2} \mathrm{O}(40 \mathrm{~mL})$, acetone $(5 \mathrm{~mL})$, solid $\mathrm{NaOH}(0.19 \mathrm{~g}, 4.8$ mmol) and $\mathrm{NaHCO}_{3}(0.4 \mathrm{~g}, 4.8 \mathrm{mmol})$ was warmed to $50{ }^{\circ} \mathrm{C}$ for $1 \mathrm{~h}$. After cooling to rt, $35 \%$ hydrogen peroxide solution $(1.9 \mathrm{~mL}, 19.2 \mathrm{mmol})$ was added dropwise. The reaction was stirred at $\mathrm{rt}$ for $24 \mathrm{~h}$, then it was shaken with EtOAc $(50 \mathrm{~mL})$ and the aqueous layer was separated and acidified to $\mathrm{pH} 4$ by dropwise addition of $2 \mathrm{~N} \mathrm{HCl}$, keeping the temperature below $5{ }^{\circ} \mathrm{C}$. Extraction with EtOAc $(2 \times 50 \mathrm{~mL})$ gave pure compound $4 \mathbf{a}$ as a white solid (1.2 g, 82\% yield), mp $205{ }^{\circ} \mathrm{C}\left(\mathrm{Et}_{2} \mathrm{O}\right)$. IR (KBr): 3410, 1701, 1449, 1329, 1157, $807 \mathrm{~cm}^{-1} .{ }^{1} \mathrm{H}$ NMR $\left(\mathrm{CDCl}_{3}\right) \delta 7.85(\mathrm{~s}, 1 \mathrm{H}, \mathrm{H} 5) ; 7.63\left(\mathrm{~d}, \mathrm{~J}_{\mathrm{H} 8-7}=8.56 \mathrm{~Hz}, 1 \mathrm{H}, \mathrm{H} 8\right) ; 7.46(\mathrm{~s}, 1 \mathrm{H}, \mathrm{OH})$; $7.09\left(\mathrm{~d}, \mathrm{~J}_{\mathrm{H} 2-3}=7.32 \mathrm{~Hz}, 1 \mathrm{H}, \mathrm{H} 2\right) ; 6.98\left(\mathrm{~d}, \mathrm{~J}_{\mathrm{H} 3-2}=7.32 \mathrm{~Hz}, 1 \mathrm{H}, \mathrm{H} 3\right) ; 6.91\left(\mathrm{~d}, \mathrm{~J}_{\mathrm{H} 7-8}=8.56 \mathrm{~Hz}, 1 \mathrm{H}, \mathrm{H} 7\right.$ ); $2.74\left(\mathrm{~s}, 3 \mathrm{H}, \mathrm{CH}_{3}\right) ; 2.43\left(\mathrm{~s}, 3 \mathrm{H}, \mathrm{CH}_{3}\right) ; 1.64\left(\mathrm{~s}, 9 \mathrm{H}, \mathrm{CH}_{3}\right)$. Anal. Calcd for $\mathrm{C}_{19} \mathrm{H}_{21} \mathrm{NO}_{3}: \mathrm{C}, 73.29 ; \mathrm{H}, 6.80 ; \mathrm{N}$, 4.50. Found: C, 73.31; H, 6.75; N, 4.50. 
Following a procedure identical to that described for compound $\mathbf{4 a}$, but using compound $\mathbf{3 b}(1.2 \mathrm{~g}, 4.8$ mmol) as starting material, compound $4 \mathbf{b}$ was obtained as a white solid (0.94 g, $70 \%$ yield), mp $202{ }^{\circ} \mathrm{C}$ $\left(\mathrm{Et}_{2} \mathrm{O}\right)$. IR (KBr): 3415, 1587, 1460, 1165, 809, $543 \mathrm{~cm}^{-1} .{ }^{1} \mathrm{H}$ NMR $\left(\mathrm{CDCl}_{3}\right) \delta 8.23\left(\mathrm{~d}, \mathrm{~J}_{\mathrm{H} 5-7}=1.96 \mathrm{~Hz}\right.$, $1 \mathrm{H}, \mathrm{H} 5) ; 7.52\left(\mathrm{dd}, \mathrm{J}_{\mathrm{H} 7-8}=8.80 \mathrm{~Hz}, 1 \mathrm{H}, \mathrm{H} 7\right) ; 7.23\left(\mathrm{dd}, \mathrm{J}_{\mathrm{H} 8-7}=8.80 \mathrm{~Hz}, 1 \mathrm{H}, \mathrm{H} 8\right) ; 7.07\left(\mathrm{~d}, \mathrm{~J}_{\mathrm{H} 2-3}=7.32 \mathrm{~Hz}\right.$, $1 \mathrm{H}, \mathrm{H} 2) ; 6.88\left(\mathrm{~d}, \mathrm{~J}_{\mathrm{H} 3-2}=7.32 \mathrm{~Hz}, 1 \mathrm{H}, \mathrm{H} 3\right) ; 4.07\left(\mathrm{~s}, 3 \mathrm{H}, \mathrm{NCH}_{3}\right) ; 2.80\left(\mathrm{~s}, 3 \mathrm{H}, \mathrm{CH}_{3}\right) ; 2.78\left(\mathrm{~s}, 3 \mathrm{H}, \mathrm{CH}_{3}\right)$. Anal. Calcd for $\mathrm{C}_{15} \mathrm{H}_{15} \mathrm{NO}$ : C, 79.97; H, 6.71; N, 6.22. Found: C, 80.00; H, 6.70; N, 6.00.

\section{5,8-Dimethyl-9H-carbazol-3-ol (5)}

Following a procedure identical to that described for compound $\mathbf{4 a}$ (1.6 g, $4.8 \mathrm{mmol})$, but reaching $\mathrm{pH} 1$ by the use of $6 \mathrm{~N} \mathrm{HCl}$ for the hydrolytic work-up, compound 5 was obtained as a white solid $(0.75 \mathrm{~g}, 75 \%$ yield), mp $174{ }^{\circ} \mathrm{C}(\mathrm{EtOH})$. IR (KBr): 3517, 3415, 1461, 1165, 847, 809, $543 \mathrm{~cm}^{-1} .{ }^{1} \mathrm{H}$ NMR (DMSO- $d_{6}$ ) $10.88(\mathrm{~s}, 1 \mathrm{H}, \mathrm{NH}) ; 9.12(\mathrm{bs}, 1 \mathrm{H}, \mathrm{OH}) ; 7.53(\mathrm{~s}, 1 \mathrm{H}, \mathrm{H} 5) ; 7.40\left(\mathrm{~d}, \mathrm{~J}_{\mathrm{H} 8-7}=8.56 \mathrm{~Hz}, 1 \mathrm{H}, \mathrm{H} 8\right) ; 7.08\left(\mathrm{~d}, \mathrm{~J}_{\mathrm{H} 2-3}=\right.$ $7.36 \mathrm{~Hz}, 1 \mathrm{H}, \mathrm{H} 2) ; 6.95$ (d, J $\left.\mathrm{J}_{\mathrm{H}-8}=8.56 \mathrm{~Hz}, 1 \mathrm{H}, \mathrm{H} 7\right) ; 6.83\left(\mathrm{~d}, \mathrm{~J}_{\mathrm{H} 3-2}=7.36 \mathrm{~Hz}, 1 \mathrm{H}, \mathrm{H} 3\right) ; 2.61\left(\mathrm{~s}, 3 \mathrm{H}, \mathrm{CH}_{3}\right)$; $2.50\left(\mathrm{~s}, 3 \mathrm{H}, \mathrm{CH}_{3}\right) .{ }^{13} \mathrm{C}$ NMR $\left(\mathrm{DMSO}-d_{6}\right) \delta 150.2,139.7,133.8,129.4,125.4,123.9,120.3,119.2,117.2$, 114.0, 111.1, 107.0, 20.1, 16.7. MS (ESI): [M+1] 212.

\section{ACKNOWLEDGMENTS}

Authors thank "Les Laboratoires Servier" and "The Conseil Régional de Basse-Normandie" for financial support.

\section{REFERENCES}

1. (a) M. Romero, P. Renard, D. H. Caignard, G. Atassi, X. Solans, P. Constans, C. Bailly, and M. D. Pujol, J. Med. Chem., 2007, 50, 294. (b) T. L. Ho and S. Y. Hsieh, Helv. Chim. Acta, 2006, 89, 111. (c) Y. C. Kuo, P. L. Kuo, Y. L. Hsu, C. Y. Cho, and C. C. Lin, Life Sci., 2006, 78, 2550. (d) N. C. Garbett and D. E. Graves, Curr. Med. Chem., 2004, 4, 149. (e) P. L. Kuo, Y. L. Hsu, Y. C. Kuo, C. H. Chang, and C. C. Lin, Anti-Cancer Drug., 2005, 16, 789. (f) P. L. Kuo, Y. L. Hsu, C. H. Chang, and C. C. Lin, Cancer Lett., 2005, 223, 293. (g) M. Hagg, M. Berdtsson, A. Mandic, R. Zhou, M. C. Shoshan, and S. Linder, Mol. Cancer Ther., 2004, 3, 489.

2. H. Chabane, C. Lamazzi, V. Thiéry, G. Guillaumet, and T. Besson, Tetrahedron Lett., 2002, 43, 2483.

3. N. Harada, T. Kawaguchi, I. Inoue, M. Ohashi, and K. Oda, Chem. Pharm. Bull., 1997, 45, 134.

4. (a) J. B. Le Pecq, N. D. Xuong, C. Gosse, and C. Paoletti, P. Natl. Acad. Sci. USA, 1974, 71, 5078. (b) A. J. F. Searle, C. Gee, and R. L. Willson, Oxygen Radicals Chem. Biol., 1984, 377.

5. (a) J. B. Le Pecq, C. Paoletti, and N. D. Xuong, Eur. Pat. Appl., 1980, EP 9445. (b) J. C. Lancelot, B. Letois, S. Rault, and M. Robba, Heterocycles, 1990, 31, 241. (c) A. Langendoen, J. P. M. Plug, G. J. 
Koomen, and U. K. Pandit, Tetrahedron, 1989, 45, 1759. (d) A. Langendoen, G. J. Koomen, and U. K. Pandit, Heterocycles, 1987, 26, 91.

6. A. S. Voisin, A. Bouillon, J. C. Lancelot, and S. Rault, Tetrahedron, 2005, 61, 1417.

7. (a) A. Cranwell and J. E. Saxton, J. Chem. Soc., 1967, 3482. (b) J. C. Lancelot, S. Rault, M. Robba, and H. D. Nguyen, Chem. Pharm. Bull., 1987, 35, 425.

8. (a) T. Tabka, B. Letois, J. C. Lancelot, P. Gauduchon, J. F. Heron, J. Y. Le Talaer, S. Rault, and M. Robba, Eur. J. Med. Chem., 1989, 24, 605. (b) A. Testard, L. Picot, I. Fruitier-Arnaudin, J. M. Piot, H. Chabane, L. Domon, V. Thiery, and T. Besson, J. Enzym. Inhib. Med. Chem., 2004, 19, 467.

9. V. Moinet-Hedin, T. Tabka, F. Sichel, P. Gauduchon, J. Y. Le Talaer, C. Saturnino, B. Letois, J. C. Lancelot, and M. Robba, Eur. J. Med. Chem., 1997, 32, 113.

10. (a) B. R. Henke, C. J. Aquino, L. S. Birkemo, D. K. Croom, R. W. Dougherty, G. N. Ervin, M. K. Grizzle, G. C. Hirst, M. K. James, M. F. Johnson, K. L. Queen, R. G. Sherrill, E. E. Sugg, E. M. Suh, J. W. Szewczyk, R. J. Unwalla, J. Yingling, and T. M. Willson, J. Med. Chem., 1997, 40, 2706. (b) V. Diep, J. J. Dannenberg, and R. W. Franck, J. Org. Chem., 2003, 68, 7907. (c) A. Kuwahara, K. Nakano, and K. Nozaki, J. Org. Chem., 2005, 70, 413. (d) M. Chakrabarty, T. Kundu, and Y. Harigaya, Synth. Commun., 2006, 36, 2069. (e) S. Routier, L. Sauge, and N. Ayerbe, Tetrahedron Lett., 2002, 43, 589.

11. R. Cao, Q. Chen, X. Hou, H. Chen, H. Guan, Y. Ma, W. Peng, and A. Xu, Bioorg. Med. Chem., 2004, 12, 4613.

12. (a) A. Bouillon, J. C. Lancelot, V. Callot, P. R. Bovy, and S. Rault, Tetrahedron, 2002, 58, 2885. (b) J. Sopkovà-de Oliveira Santos, J. C. Lancelot, A. Bouillon, and S. Rault, Acta Crystallogr. C, 2003, 59, o111.

13. A. Bouillon, J. C. Lancelot, V. Callot, P. R. Bovy, and S. Rault, Tetrahedron, 2002, 58, 3323.

14. A. Bouillon, J. C. Lancelot, V. Callot, P. R. Bovy, and S. Rault, Tetrahedron, 2002, 58, 4369.

15. (a) A. Bouillon, J. C. Lancelot, J. Sopkovà-de Oliveira Santos, V. Callot, P. R. Bovy, and S. Rault, Tetrahedron, 2003, 59, 10043. (b) J. Sopkovà-de Oliveira Santos, A. Bouillon, J. C. Lancelot, and S. Rault, Acta Crystallogr. C, 2003, 59, 0596. 